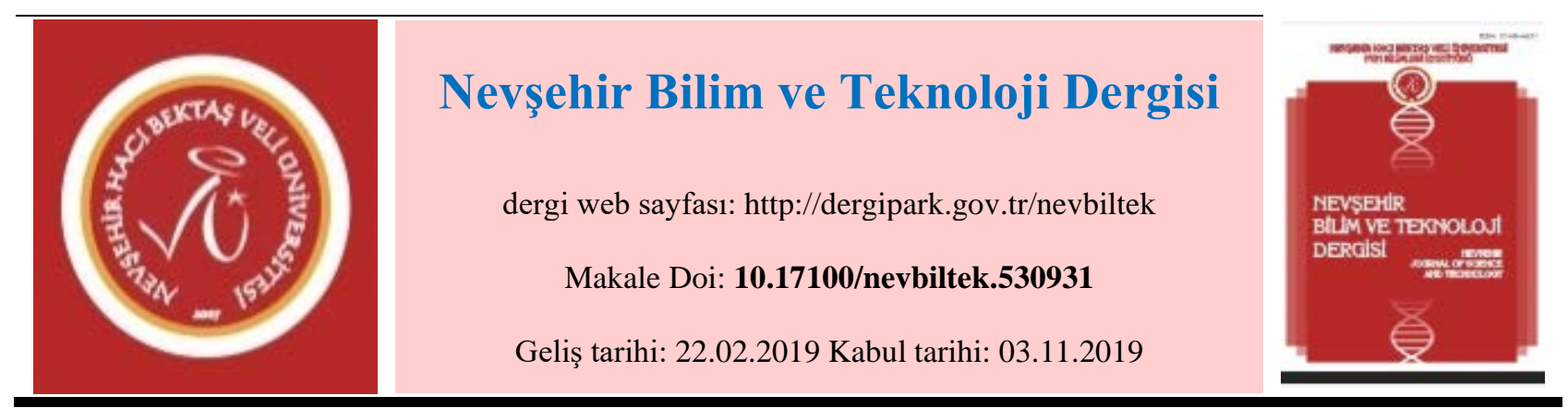

\title{
2-Sülfoterfitalik Asit ve 4,4'-Bipiridin İçeren 2-Boyutlu Koordinasyon Bileşiğinin Yapısal ve Optik Özellikleri
}

\author{
Mustafa Burak ÇOBAN ${ }^{1 *}$ ve Hülya KARA SUBAŞAT ${ }^{2}$ \\ ${ }^{1 *}$ Ballkesir Üniversitesi, Bilim ve Teknoloji Uygulama ve Araştırma Merkezi, Ballkesir \\ ORCID ID: http://orcid.org/0000-0003-3488-5284 \\ ${ }^{2}$ Muğla Sttkı Koçman Üniversitesi, Fen Bilimleri Enstitüsü, Enerji Bölümü, Muğla \\ ORCID ID: http://orcid.org/ 0000-0002-2032-8930
}

$\ddot{O} \mathbf{z}$

$\mathrm{Bu}$ çalışmada, iki farklı organik ligand içeren yeni bir tip iki-boyutlu koordinasyon bileşiği, $\left\{\left(2-\mathrm{H}_{3} \mathrm{stp}\right)\left(4,4^{\prime}-\text {-bipy }\right)_{2} \text {. }\left(\mathrm{H}_{2} \mathrm{O}\right)\right\}_{\mathrm{n}},\left(2-\mathrm{H}_{3} \mathrm{Stp}=2\right.$-sülfoterfitalik asit ve 4,4'-bipy= 4,4'-bipiridin), hidrotermal yöntemle sentezlendi ve tek kristal X ışını kristalografisi, toz kırınımı, UV-Vis spektroskopisi ve katı hal fotolüminesans ölçümleri ile karakterize edildi. Kristal yapı analizi bileşiğin triklinik kristal sistemi P-1 uzay grubunda kristalleştiğini gösterir. Bileşiğin asimetrik birimi bir 2-sülfoterfitalik asit, iki 4,4'-bipiridin ve bir örgü su moleküllerinden oluşmaktadır. Kristal yapıda her bir 2- $\mathrm{H}_{3}$ stp ligandı güçlü O$\mathrm{H} \cdots \mathrm{N}$ hidrojen bağları ile 4,4'-bipiridin molekülü ile etkileşim içindedir. Yapı böylece tek-boyutlu zincir şeklinde büyümektedir. Ayrıca, O-H $\cdots \mathrm{O}, \mathrm{O}-$ $\mathrm{H} \cdots \mathrm{N}$ hidrojen bağları ve yüz yüze $\pi-\pi$ etkileşimler ile molekülleri bir arada tutmaktadır. Sonuç olarak yapı iki-boyutlu bir forma sahip olur. Ayrıca, bileşik ve onun ligandları sırasıyla oda sıcaklığında katı halde şiddetli sarı-yeşil ve yeşil lüminesans sergiler. Bileşik, maksimum dalgaboyu $560 \mathrm{~nm}$ de olacak şekilde geniş yayma bandı gösterir. Bu geçiş muhtemelen $\pi-\pi *$ veya $n-\pi *$ ligandlar arası elektronik geçiş olabilir. Bu arada, bileşiğin ölçülen fotolüminesans spektrumunda, serbest ligandlarla karşılaştırıldığında yaklaşı $50 \mathrm{~nm}$ kırmızıya kayma gözlenmiştir. Böylece, bileşik sarımsı-yeşil ışık yayar. Bileşik, $\left\{\left(2-\mathrm{H}_{3} \mathrm{stp}\right)\left(4,4^{\prime}-\text {-bipy }\right)_{2} \text {. }\left(\mathrm{H}_{2} \mathrm{O}\right)\right\}_{\mathrm{n}}$, hem teknolojik hem de endüstriyel üretimde yapısal bir ara malzeme olarak kullanılabilir.

Anahtar Kelimeler: 2-sülfoterfitalik asit, 4,4'-bipiridin, Koordinasyon bileşiği, Tek kristal analizi, Fotolüminesans özellikleri

\section{Structural and Optical Properties of 2-Dimensional Coordination Compound Containing 2- Sulfoterephthalic Acid and 4,4'-Bipyridine}

\begin{abstract}
In this study, a new type 2D coordination compound formulated as $\left\{\left(2-\mathrm{H}_{3} \mathrm{stp}\right)\left(4,4^{\prime}-\text {-bipy }\right)_{2} \text {. }\left(\mathrm{H}_{2} \mathrm{O}\right)\right\}_{\mathrm{n}},\left(2-\mathrm{H}_{3} \mathrm{stp}=2\right.$-sulfoterephthalic acid and 4,4'-bipy= 4,4'-bipyridine), which included two different organic ligand, was synthesized via hydrothermal method and characterized by single crystal X-ray crystallography, powder X-ray diffraction (PXRD), UV-vis and solid-state photoluminescence measurements. The crystal structure analysis shows that compound crystallizes in the triclinic space group P-1 and its asymmetric unit of contains one 2-sulfoterephthalic acid, two 4,4'-bipyridine and one coordinated water molecules. In this structure, each 2- $\mathrm{H}_{3}$ stp ligand links to one $4,4^{\prime}$-bipy molecule with $\mathrm{O}-\mathrm{H} \cdots \mathrm{N}$ hydrogen bonds and the structure is expanding in the form of one-dimensional chain. Meanwhile, $\mathrm{O}-\mathrm{H} \cdots \mathrm{O}, \mathrm{O}-\mathrm{H} \cdots \mathrm{N}$ hydrogen bonds and face-to-face $\pi-\pi$ interactions contact also connect the molecules in the structure, resulting form to 2D. Furthermore, compound and its ligand display an intense yellow and green light luminescence emission in the solid-state at room temperature, respectively. The compound indicates broad emission band at $\lambda_{\max }=560 \mathrm{~nm}$ which may be assigned to the $\pi-\pi^{*}$ or $n-\pi^{*}$ electronic transition intra-ligand charge transfer (ILCT). Meanwhile, the photoluminescent measured of compound shows the about 50 $\mathrm{nm}$ red shift compared free ligands. Thus, compound emits yellowish-green light. The compound, $\left\{\left(2-\mathrm{H}_{3} \mathrm{stp}\right)\left(4,44^{\prime}-\mathrm{bipy}\right)_{2} \text {. }\left(\mathrm{H}_{2} \mathrm{O}\right)\right\}_{\mathrm{n}}$, can be used as a structural intermediate material in both technological and industrial production.
\end{abstract}

Keywords: 2-sulfoterephthalic acid, 4,4'-bipyridine, Coordination compound, Single crystal analysis, Photoluminescence properties 
Nevşehir Bilim ve Teknoloji Dergisi (2019), 8(2) 103-113

\section{Giriş}

Son yirmi yılda koordinasyon bileşiklerinin gösterdiği moleküler tanıma, iyon değişimi, katalizleme, lüminesans, gaz adsorpsiyonu ve ayırma süreçleri, lineer olmayan optik alanlarında çeşitlilik gösteren ilginç mimarileri ve potansiyel uygulamaları nedeniyle bilimsel çevrede büyük ilgi görmektedir [1-6].

Son zamanlarda yapılan araştırmalar, moleküler yapıların, moleküller arası hidrojen $(H)$, nitrojen $(N)$ ve oksijen (O) bağlanma gibi özel etkileşimler sonucunda yapısal ve elektronik özelliklerinde önemli değişikler meydana geldiği gözlemlenmiştir [7-9]. Bu etkileşimler içerisinde, özellikle, N-verici ve O-verici karboksilat içeren ligandlar, koordinasyon bileşiklerinin oluşumunda yüksek kararlılık ve sahip oldukları potansiyel uygulamaları nedeniyle tercih edilmiştir [10,11]. 2-sülfoterfitalik asid ve türevleri iki karboksilat ve bir adet sülfat grubu içermektedir. Bu fonksiyonel gruplar yüksek boyutlu ve farklı mimarili koordinasyon bileşiklerinin oluşumuna olanak sağlamaktadır. Ayrıca sülfat gruplarının zayıf etkileşim yetenekleriyle, koordinasyon modlarına esneklik ve daha büyük bir koordinasyon çeşitliliği sağlamaktadır ve yapısal çeşitlilik kaynağı olan kimyasal ortama duyarlı hale getirebilir [12,13].

Bununla birlikte, biyomedikal uygulamalara, farklı mimarileri, kalıcı yüksek ve düzenli gözenekliliği, iyi biyouyumlu ve çok yönlü işlevselliği nedeniyle ilaç molekülü depolanması ve yüzeysel dağıtıcı özelliklerinin nedeniyle koordinasyon bileşikleriyle çalışmalar denenmiştir. Ayrıca, koordinasyon polimerli-ilaç sistemlerinde, hidrojen bağları, elektrostatik etkileşim ve $\pi$ - $\pi$ etkileşimi gibi etkileşimler, kontrol edilebilir ve silindirik ilaç salınımını başarmak için önemli olan ilacın yayılmasını ve salınmasını düzenler [14,15].

Son birkaç yıldır, araştırma grubumuz çeşitli aromatik karboksilat ve piridin türevleri içeren organik yapılı moleküllerde yapısal ve optik karakterizasyon konularında aktif olarak çalışmıştır [16-18]. Bu çalışmada, daha önceki çalışmalarımızın devamı niteliğinde yeni $\left\{\left(2-\mathrm{H}_{3} \mathrm{stp}\right)(4,4 \text { '-bipy })_{2} \text {. }\left(\mathrm{H}_{2} \mathrm{O}\right)\right\}_{\mathrm{n}}\left(2-\mathrm{H}_{3} \mathrm{stp}=2\right.$-sülfoterfitalik asit, 4,4'-bipy=4,4'bipiridin) bileşiği sentezlendi ve tek kristal X-ışını kırınım yöntemi ile karakterize edildi. Ayrıca kristal yapıdaki molekül içi ve moleküler arası $\mathrm{O}-\mathrm{H} \cdots \mathrm{N}$, O-H$\cdots \mathrm{O}$ ve $\mathrm{C}-\mathrm{H} \cdots \mathrm{O}$ hidrojen bağları ve $\pi-\pi$ etkileşimleri incelendi. Bunlara ilaveten UV ve görünür bölge soğurma spektrumları hem bileşik hem de bileşiğin temel yapısını oluşturan serbest ligandlar için karşılaştırması yapıldı. Son olarak ise hem bileşiğin hem de bileşiği oluşturan organik ligandların görünür bölgede fotolüminesans spektrumları detaylı olarak incelenmiştir.

\section{Materyal ve Metot}

Deneyde kullanılan kimyasal maddeler ve çözücüler TCI-America ve Sigma-Aldrich firmasından ticari olarak alınmıştır ve herhangi bir saflaştırma işlemi uygulamadan kullanılmıştır. Kompleksin element analizi LECO-CHNS-932 marka cihaz ve Tek kristal $\mathrm{X}$-1şınları analizi CrysAlis ${ }^{\text {Pro }}$ yazılımına sahip EOS-CCD detektörü ile Rigaku-Oxford Diffraction Xcalibur X-Işınları Tek Kristal Difraktometre cihazı ile Mo-Ka ışını kullanılarak oda sıcaklığında ölçülmüş̧ür [19]. Bileşiğin kristal yapısı, direk yöntem kullanılarak SHELXS arayüzü ile OLEX2 programıyla çözülmüştür [20,21]. Hidrojen dışındaki bütün atomlar anizotropik yer değiştirme parametresi olarak tanımlandı ve konumsal kısıtlama olmaksızın revize edildi. Hidrojen atomları ideal bir şekilde konumlandırılarak izotropik yer değiştirme parametresi kendilerine bağlı olan karbon atomlarının 1.2 katına sınırlandıııldı. Moleküller arası $\pi$ etkileşimleri ise detaylı bir şekilde PLATON 1.17 programı ile hesaplandı [22]. Katı hal soğurma spektrumları Ocean Optics Maya 2000 Pro UV-Vis spektrometre ile kaydedildi. Fotolüminesans spektrumu Andor Solis SR 500i-BL model spektrometre ile oda sıcaklı̆̆ında elde edildi. 


\section{$2.1\left\{\left(2-H_{3} s t p\right)\left(4,4^{\prime}-\text { bipy }\right)_{2 .}\left(\mathrm{H}_{2} \mathrm{O}\right)\right\}_{\mathrm{n}}$ Bileşiğinin Sentezlenmesi}

2-sülfoterfitalik asit sodyum tuzu (1 mmol) ve 4,4'-bipiridin (1 mmol), 23 ml $\mathrm{H}_{2} \mathrm{O}$ çözeltisinde 60 dakika oda sıcaklığında manyetik karıştırıcı ile karıştırılmıştır. Bu karışım kaynama noktasına kadar isıtılarak çözülmüş ve bu çözeltiye $5 \mathrm{ml} \mathrm{NaOH}$ eklenerek pH değeri: 4,02 değerine yükseltilerek, çözelti 30 dakika daha kaynama sıcaklı̆̆ında manyetik karıştırıı ile karıştııılmıştır. Elde edilen çözelti daha sonra teflon kaplı bir otoklava aktarılmıştır. Reaktör, oda sicaklığından $120^{\circ} \mathrm{C}$ dereceye kadar 10 saatte isıtılıp, 60 saat $120^{\circ} \mathrm{C}$ sıcaklıkta tutulmuş ve 48 saatte oda sicaklığına düşecek şekilde programlanan etüve yerleştirilmiştir. Etüvden alınan çözelti yaklaşı üç hafta boyunca karanlıkta ve oda sıcaklığında bekletilmiş ve renksiz prizmatik tek kristaller süzme kâğıdıyla ayrıştırılmıştır. Verim \% 79. Teorik element analizi (\%) $\left(\mathrm{C}_{28} \mathrm{H}_{24} \mathrm{~N}_{4} \mathrm{O}_{8} \mathrm{~S}\right)$ : C:58.33, H:4.27, N:16.89; Deneysel element analizi (\%): C:58,31, H:4.26, N:16.95.

\section{Bulgular}

\subsection{Tek Kristal Yapı Analizi}

Sentezlenen bileşiğin kristallografik verileri ve yapı arıtım parametreleri Tablo 1 de listelenmektedir. Seçilmiş bağ uzunlukları ve açıları Tablo 2 de özetlenmiştir. Molekül içi ve moleküller arası $\mathrm{O}-\mathrm{H} \cdots \mathrm{N}$, O-H $\cdots \mathrm{O}$ ve C-H $\cdots \mathrm{O}$ hidrojen bağı geometrisi $\left(\AA,{ }^{\circ}\right)$ ve aromatik halkaların merkezleri arasındaki uzaklıklar ile $\pi$ - $\pi$ etkileşimleri Tablo 3 de verilmiştir. Bileşiğin moleküler yapısı Şekil 1 de, paketlenmiş yapısı Şekil 2 ve 3 de aromatik halkalar arasındaki $\pi-\pi$ etkileşimleri ise Şekil 4 de gösterilmiştir.

Bileşik, triklinik kristal sistemi P-1 uzay grubunda kristalleşmiştir. Bileşiğin asimetrik birimi, 1:2:1 oranında 2sülfoterfitalik asit, 4,4'-bipiridin ve örgü su moleküllerinden oluşmaktadır. Şekil 1' de görüldüğü gibi, kristal yapı bir adet $\mathrm{H}_{3}$ stp ligandının karboksil grupları güçlü hidrojen bağları ile hem örgü su molekülüne hem de 4,4'-bipiridin molekülünün azot atomu ile etkileşim halindedir. Kristal yapıda, moleküller güçlü molekül içi ve moleküler arası $\mathrm{O}-\mathrm{H} \cdots \mathrm{N}, \mathrm{O}-\mathrm{H} \cdots \mathrm{O}$ hidrojen bağları ile bir arada tutulur ve $\mathrm{C}-\mathrm{H} \cdots \mathrm{O}$ zayıf hidrojen bağı ile daha kararlı hal alır (Şekil 2). 2-sülfoterfitalik asit ve 4,4'-bipiridin aromatik grupları arasındaki güçlü $\mathrm{O}-\mathrm{H}^{\cdots} \cdots \mathrm{N}$ hidrojen bağları ile yapı bir boyutlu zincir şeklinde büyümektedir. Ayrıca, hidrojen bağlı moleküler yapı $\mathrm{C}-\mathrm{H} \cdots \mathrm{O}$ hidrojen bağları ve aromatik halkalar arasındaki $\pi-\pi$ etkileşimleri ile iki-boyutlu tabakalı yapıyı oluşturmaktadır (Şekil 3). 2-sülfoterfitalik asit ve 4,4'-bipiridin aromatik halkalarının merkezleri arasında 4.0482(14)-5.0799(14) Å' luk mesafeler ile $\pi-\pi$ etkileşimleri gözlenmiştir (Şekil 4).

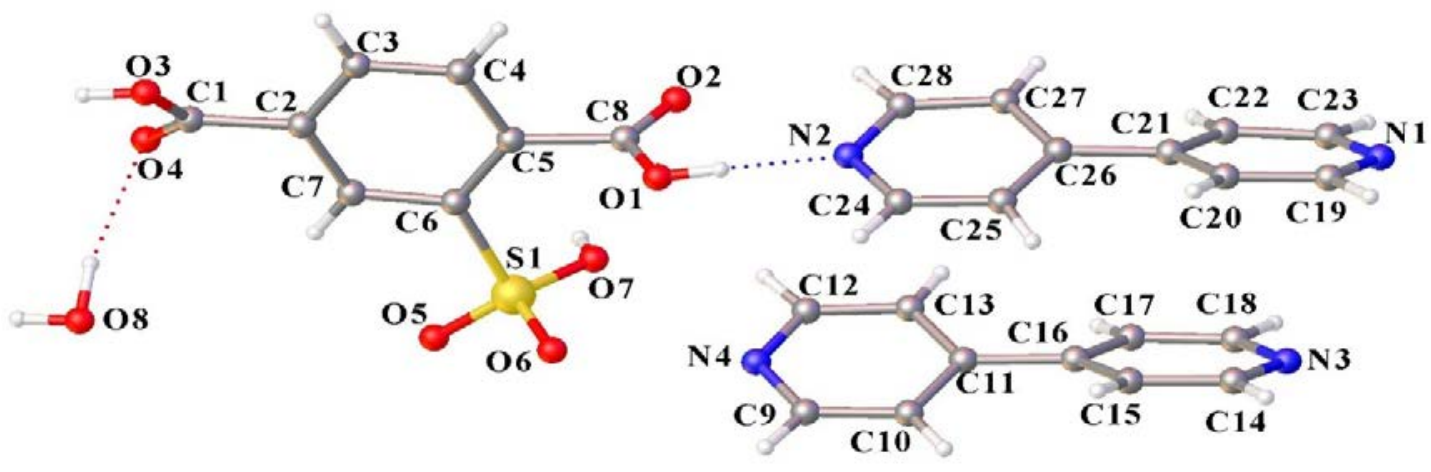

Şekil 1. Bileşiğin moleküler yapısı. 
Nevşehir Bilim ve Teknoloji Dergisi (2019), 8(2) 103-113

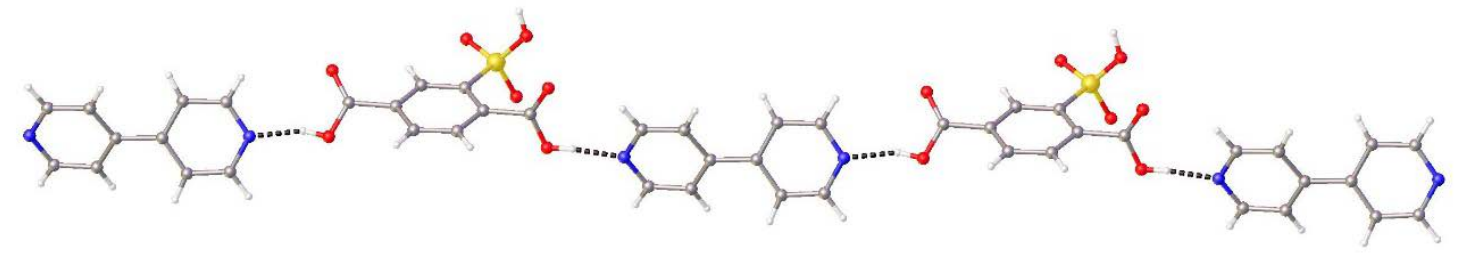

Şekil 2. Bileşiğin $\mathrm{O}-\mathrm{H} \cdots \mathrm{N}$ güçlü hidrojen bağları ile büyütülmüş bir-boyutlu zincir görünümü.

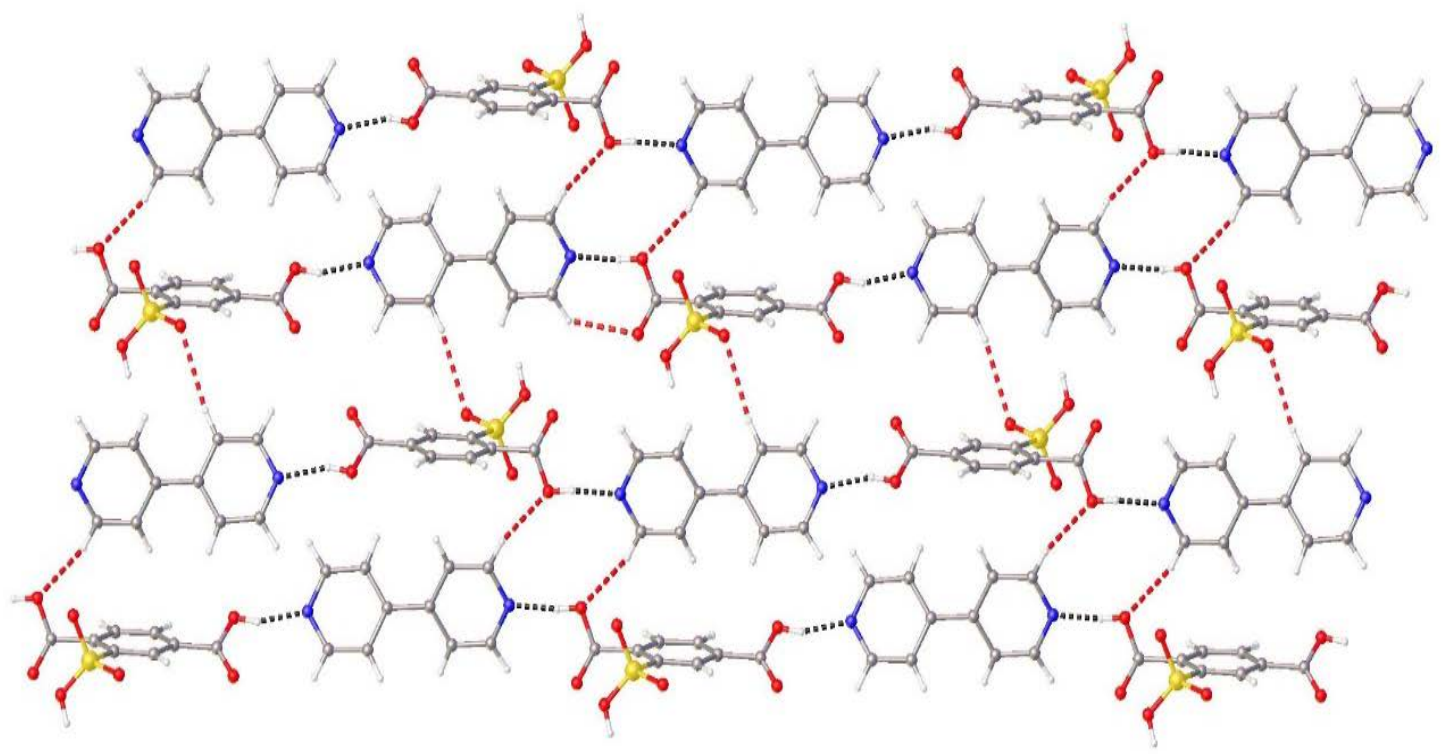

Şekil 3. Bileşiğin hidrojen bağları ile büyütülmüş iki-boyutlu tabakalı yapısı.

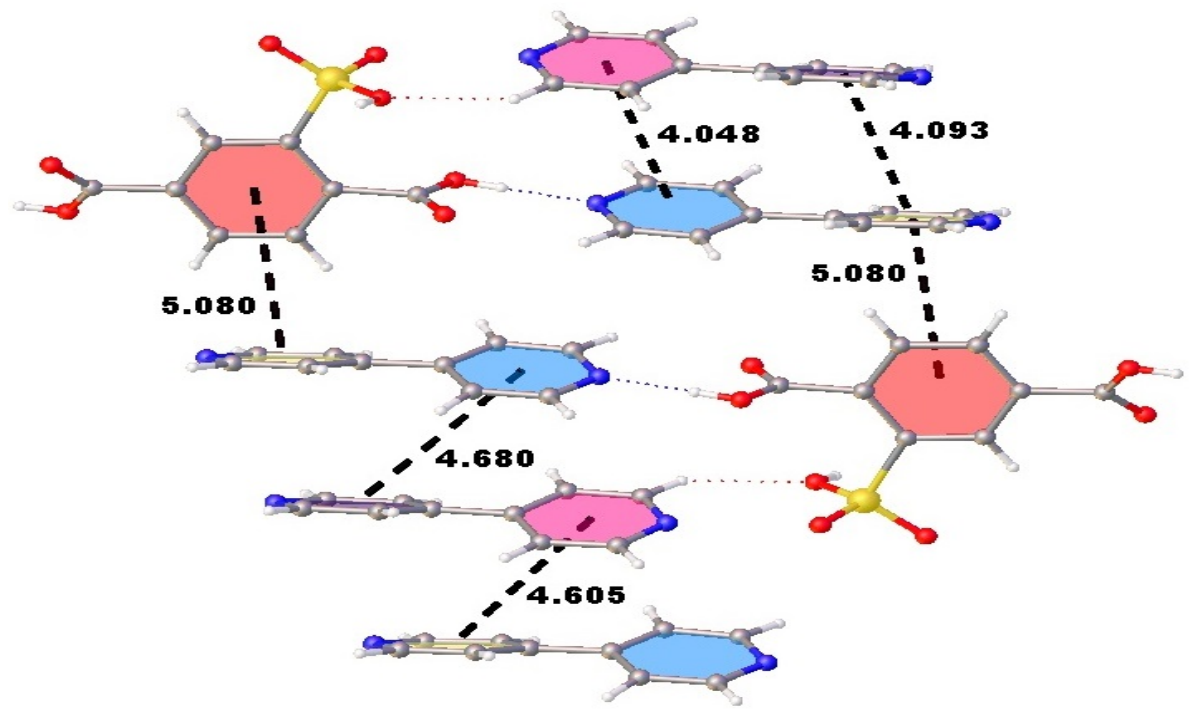

Şekil 4. Kristal yapıdaki $\pi-\pi$ etkileşmesinin görünümü. 
Nevşehir Bilim ve Teknoloji Dergisi (2019), 8(2) 103-113

Tablo 1. Moleküle ait kristalografik bilgiler ve deneysel veriler.

\begin{tabular}{ll}
\hline Molekülün Kapalı Formülü & $\mathrm{C}_{28} \mathrm{H}_{24} \mathrm{~N}_{4} \mathrm{O}_{8} \mathrm{~S}$ \\
Molekülün Ağırlığı $\left(\mathrm{g} \mathrm{mol}^{-1}\right)$ & 576.57 \\
Kristal Sistemi, Uzay Grubu & Triclinic, P-1 \\
Sıcaklık (K) & 293 \\
Birim Hücre Parametreleri & $7.7981(4), 10.4273(6), 17.3745(11)$ \\
$a, b, c(\AA)$ & \\
$\alpha, \beta, \gamma\left({ }^{\circ}\right)$ & $74.674(5), 82.980(5), 72.632(5)$ \\
Birim Hücre Hacmi $\left(\AA^{3}\right)$ & $1298.97(14)$ \\
Birim Hücredeki Molekül Sayısı & 2 \\
$\mu\left(\mathrm{mm}^{-1}\right)$ & 0.186 \\
Ölçülen Yansıma & 7954 \\
Bağımsı Yansıma & 5234 \\
Gözlenen Yansıma Sayıları & 4064 \\
$R_{\text {int }}$ & 0.017 \\
$R\left[F^{2}>2 \sigma\left(F^{2}\right)\right], w R\left(F^{2}\right), S$ & $0.0478,0.1253,1.027$ \\
İncelenen Parametre Sayısı & 466 \\
$\Delta \rho_{\max }, \Delta \rho_{\min }\left(\mathrm{e} \AA^{-3}\right)$ & $0.48 /-0.33$ \\
\hline
\end{tabular}

Tablo 2. Seçilen geometrik parametreler $\left(\AA,{ }^{\circ}\right)$.

\begin{tabular}{llll}
\hline O1-C8 & $1.287(2)$ & N1-C19 & $1.330(3)$ \\
O2-C8 & $1.203(2)$ & N1-C23 & $1.323(3)$ \\
O3-C1 & $1.290(3)$ & N2-C24 & $1.332(3)$ \\
O4-C1 & $1.214(3)$ & N2-C28 & $1.333(3)$ \\
O5-S1 & $1.4372(16)$ & N3-C14 & $1.323(3)$ \\
O6-S1 & $1.4604(15)$ & N3-C18 & $1.325(3)$ \\
O7-S1 & $1.4391(16)$ & N4-C9 & $1.334(3)$ \\
C6-S1 & $1.790(2)$ & N4-C13 & $1.336(3)$ \\
O1-C8-O2 & $125.15(19)$ & O6-S1-O7 & $111.79(10)$ \\
O1-C8-C5 & $113.37(16)$ & O7-S1-C6 & $106.58(9)$ \\
O2-C8-C5 & $121.31(19)$ & N1-C19-C20 & $122.7(2)$ \\
O3-C1-O4 & $124.1(2)$ & N1-C23-C22 & $123.4(2)$ \\
O3-C1-C2 & $113.9(2)$ & N2-C24-C25 & $122.39(19)$ \\
O4-C1-C2 & $122.0(2)$ & N2-C28-C27 & $122.8(2)$ \\
O5-S1-O6 & $112.45(10)$ & N3-C14-C15 & $123.9(2)$ \\
O5-S1-O7 & $114.77(10)$ & N3-C18-C17 & $124.2(2)$ \\
O5-S1-C6 & $105.55(10)$ & N4-C9-C10 & $120.7(2)$ \\
O6-S1-C6 & $104.79(9)$ & N4-C13-C12 & $120.9(2)$ \\
\hline
\end{tabular}


Nevşehir Bilim ve Teknoloji Dergisi (2019), 8(2) 103-113

Tablo 3. Hidrojen bağları $\left(\AA,^{\circ}\right), \mathrm{Cg}-\mathrm{Cg}$ mesafeleri ile kısa halka etkileşmeleri.

\begin{tabular}{|c|c|c|c|c|c|}
\hline D-H $\cdots \mathbf{A}^{*}$ & D-H & $\mathbf{H} \cdots \mathbf{A}$ & $\mathbf{D} \cdots \mathbf{A}$ & D-H $\cdots A$ & Simetri Kodu \\
\hline $\mathrm{O} 1-\mathrm{H} 1 \cdots \mathrm{N} 2$ & 0.84 & 1.73 & 2.570 & 175 & - \\
\hline O3-H3 $\cdots \mathrm{N} 1$ & 0.84 & 1.79 & 2.604 & 161 & $-1+x, 1+y,-1+z$ \\
\hline O8-H8A $\cdots \mathrm{O} 4$ & 0.95 & 2.02 & 2.866 & 148 & - \\
\hline O8-H8B $\cdots \mathrm{N} 3$ & 0.83 & 2.16 & 2.960 & 165 & $-1+x, 1+y,-1+z$ \\
\hline СЗ-Н3А $\cdots$ O8 & 0.93 & 2.54 & 3.386 & 151.5 & $1+\mathrm{x}, \mathrm{y}, \mathrm{z}$ \\
\hline $\mathrm{C} 4-\mathrm{H} 4 \cdots \mathrm{O} 5$ & 0.96 & 2.48 & 3.391 & 158.8 & $1+x, y, z$ \\
\hline C9-H9 $\cdots$ O5 & 0.96 & 2.55 & 3.435 & 154 & $-x, 2-y, 1-z$ \\
\hline C9-H9 $\cdots$ O6 & 0.96 & 2.51 & 3.301 & 140 & $-x, 2-y, 1-z$ \\
\hline $\mathrm{C} 12-\mathrm{H} 12 \cdots \mathrm{O} 2$ & 0.90 & 2.60 & 3.151 & 121 & 1-x,1-y,1-z \\
\hline $\mathrm{C} 12-\mathrm{H} 12 \cdots \mathrm{O} 7$ & 0.90 & 2.46 & 3.255 & 148 & 1-x,1-y,1-z \\
\hline 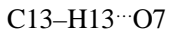 & 0.94 & 2.39 & 3.185 & 143 & - \\
\hline C14-H14으 & 0.95 & 2.56 & 3.380 & 145 & 1-x,2-y,1-z \\
\hline C24-H24 $\cdots \mathrm{O} 1$ & 0.92 & 2.48 & 3.374 & 162 & $1-x, 2-y, 1-z$ \\
\hline 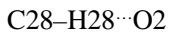 & 0.96 & 2.57 & 3.264 & 129.1 & - \\
\hline $\mathrm{Cg}(\mathrm{I}) \ldots \mathrm{Cg}(\mathrm{J})$ & & & $\mathrm{Cg}-\mathrm{Cg}$ & & \\
\hline $\operatorname{Cg}(1) \ldots \operatorname{Cg}(2)$ & & & $5.0799(14)$ & & 2-x,1-y,1-z \\
\hline $\operatorname{Cg}(1) \ldots \operatorname{Cg}(3)$ & & & 4.9831(12) & & 1-x,2-y,1-z \\
\hline $\operatorname{Cg}(1) \ldots \operatorname{Cg}(4)$ & & & 4.9501(13) & & 1-x,1-y,1-z \\
\hline $\operatorname{Cg}(2) \ldots \operatorname{Cg}(4)$ & & & 4.0933(15) & & $1+\mathrm{x}, \mathrm{y}, \mathrm{z}$ \\
\hline $\operatorname{Cg}(2) \ldots \operatorname{Cg}(5)$ & & & $4.6048(14)$ & & $1+x, y, z$ \\
\hline $\operatorname{Cg}(3) \ldots \operatorname{Cg}(4)$ & & & $4.6805(14)$ & & $\mathrm{x}, \mathrm{y}, \mathrm{z}$ \\
\hline Cg(3)...Cg(5) & & & $4.0482(14)$ & & $1+\mathrm{x}, \mathrm{y}, \mathrm{z}$ \\
\hline $\operatorname{Cg}(4) \ldots \operatorname{Cg}(4)$ & & & $5.0623(14)$ & & 1-x,1-y,2-z \\
\hline
\end{tabular}

\subsection{Toz kristal X-ışını ölçümü (PXRD):}

Şekil 5' de bileşiğin deneysel toz kırınımı deseni ve tek kristal ölçümünden simule edilmiş modeli verilmektedir. Elde edilen tek kristal örneğin kırınım pikleri ile toz kırınım analizinden elde edilen kırınım pikleri ile konum ve pik desenleri bakımından hafif kaymalar ile birlikte uyum içerisinde olduğu gözlenmektedir.

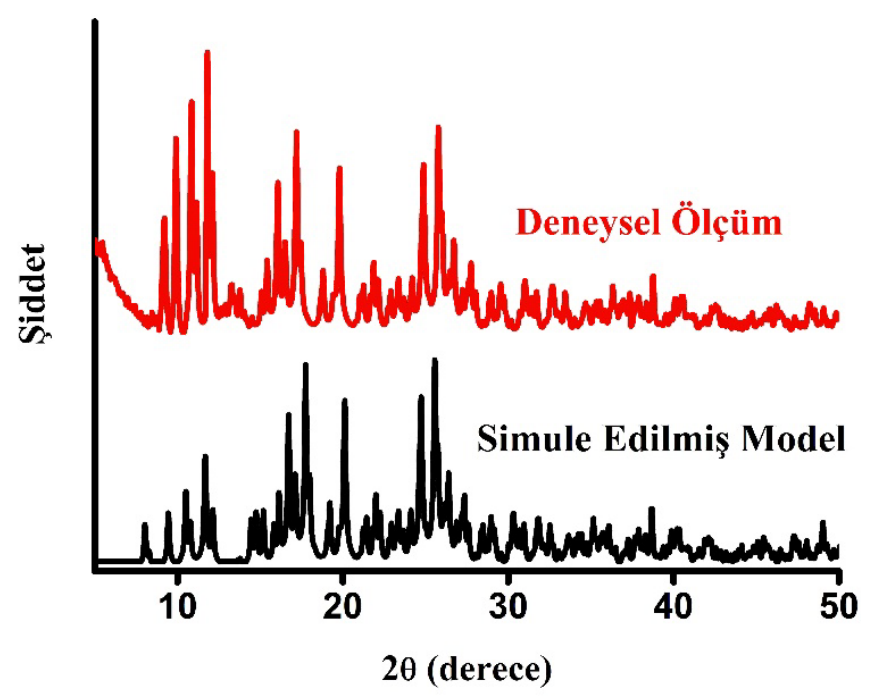

Şekil 5. Bileşiğin toz kırınım desenleri. Deneysel ölçüm (Kırmızı) ve tek kristal ölçümünden simule edilmiş model (Siyah).

\subsection{UV-Vis Soğurma Spektroskopisi}


Nevşehir Bilim ve Teknoloji Dergisi (2019), 8(2) 103-113

Bileşik ve bileşiği oluşturan serbest 2-sülfoterfitalik asit ve 4,4'-bipiridin organik ligandlarının oda sıcaklığında katı-hal UV ve görünür bölge soğurma spektrumlarının karşılaştırması Şekil 6' da verilmiştir. Serbest 2-sülfoterfitalik asit ve 4,4'-bipiridin organik ligandlarının soğurma spektrumları incelendiğinde 2-sülfoterfitalik asit ligandı merkezi $377 \mathrm{~nm}$ dalgaboyu değerine ulaşan geniş bir soğurma bandı içerirken, 4,4'-bipiridin ligandının merkezleri 282 ve $484 \mathrm{~nm}$ dalgaboyu değerinde olan iki soğurma bandı görülmektedir. Elde edilen soğurma bantları muhtemelen n- $\pi^{*}$ veya $\pi$ - $\pi^{*}$ elektronik geçişi olabilir. Bileşiğin soğurma spektrumu incelendiğinde $338 \mathrm{~nm}$ de orta şiddetli ve $423 \mathrm{~nm}$ şiddetli iki soğurma bandı gözlemlenmiştir. $338 \mathrm{~nm}$ de gözlenen yüksek enerji bandı büyük ihtimalle ligand kaynaklı n- $\pi^{*}$ veya $\pi$ $\pi^{*}$ geçişi olabilirken, diğer şiddetli band ise ligandlar arası yük geçişinin sağlandığı enerji transfer bandı olabilir [23-26].

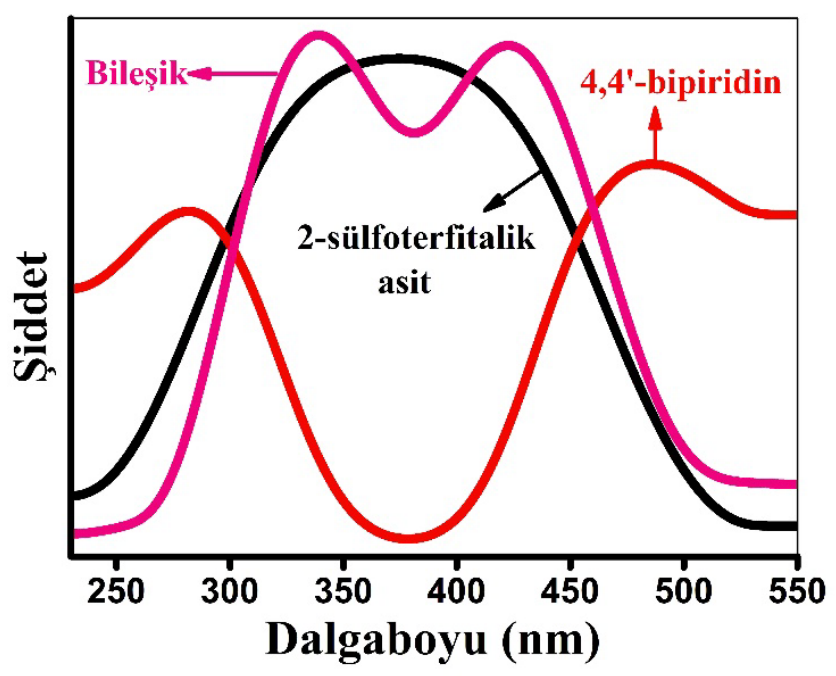

Şekil 6. Bileşik ve bileşiği oluşturan serbest 2-süfoterfitalik asit ve 4,4'-bipiridin ligandlarının UV-görünür bölge soğurma spektrumları.

\subsection{Fotolüminesans Spektroskopisi}

Bileşik ve bileşiği oluşturan organik ligandın yayma spektrumları katı-halde ve oda sıcaklığında $\lambda=349 \mathrm{~nm}$ lazer uyarması uygulanarak elde edilmiştir (Şekil 7). 2-süfoterfitalik asit ve 4,4'-bipiridin organik ligandları geniş yayma bantları göstermiştir. Bu geniş yayma bantları 2-süfoterfitalik asit için maksimum dalgaboyu $480 \mathrm{~nm}, 510 \mathrm{~nm}$ ve $558 \mathrm{~nm}$ olacak şekilde üç tepe noktası elde edilirken, 4,4'-bipiridin için ise maksimum dalgaboyu $512 \mathrm{~nm}$ de merkezlenmiş tek bir geniş yayma bandı gözlenmiştir [27,28]. Her iki organik ligandın maksimum dalgaboyu değerleri elektromanyetik spektrumun yeşil bölgesi içerisinde yer almaktadır. Ligand tabanlı bileşikler genellikle keskin pikler yerine geniş yayma bantları içermektedir. Bileşiğin yayma spektrumu incelendiğinde maksimum dalgaboyu $560 \mathrm{~nm}$ de merkezlenmiş geniş yayma bandı gözlenmiştir ve bu dalgaboyu elektromanyetik spektrumun yeşil-sarı bölgesi içerisinde yer almaktadır. Bu geçiş muhtemelen ligandlar arası yük transferi nedeniyle $n-\pi^{*}$ veya $\pi$ - $\pi^{*}$ geçişleri olabilir. Bileşik ve bileşiği oluşturan organik ligandlar karşılaştıııldığında yayma bantlarının maksimum dalgaboyları arasında yaklaşık olarak $50 \mathrm{~nm}$ lik kırmıza kayma ortaya çıkmaktadır. Ortaya çıkan bu kayma kompleksleşme sırasında meydana gelen elektronik enerji transferi ve bileşiğin mikro-çevresinden kaynaklanmaktadır. Böylece bileşik güçlü yeşil-sarı 1şıma yapmaktadır. 


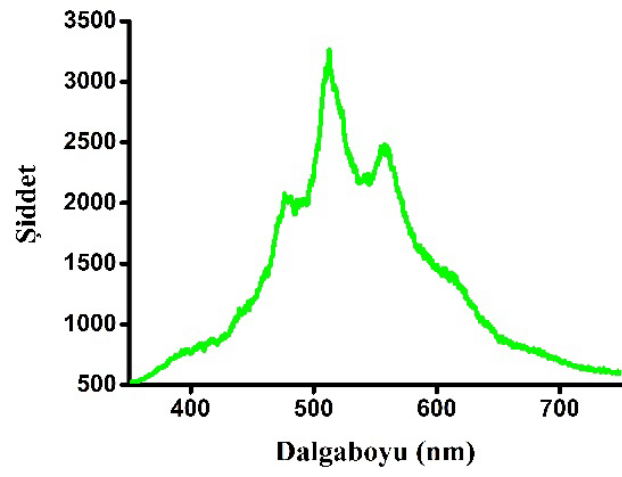

(a)

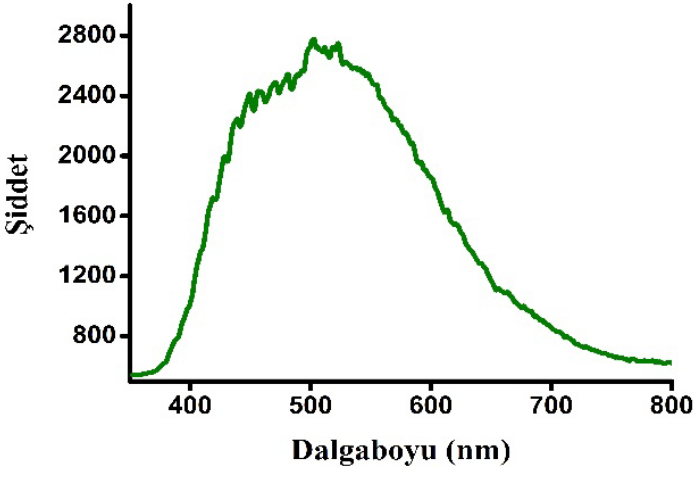

(b)

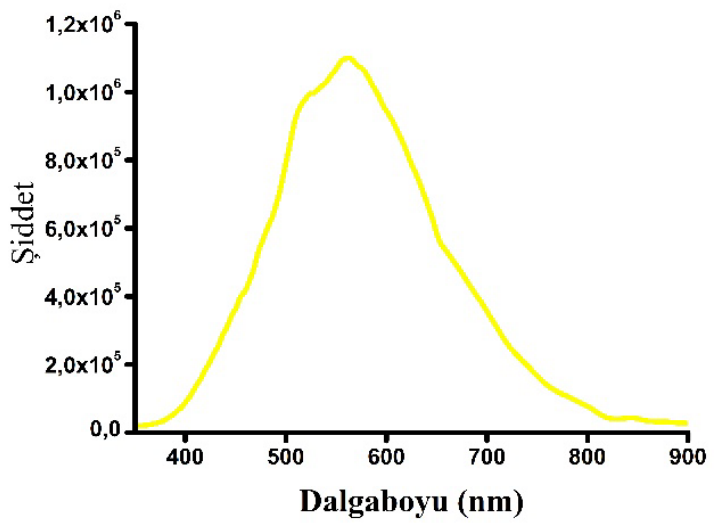

(c)

Şekil 7. Oda sıcaklığında, katı-halde fotolüminesans spektrumları ( $\lambda=349 \mathrm{~nm})$. a) 2-sülfoterfitalik asit ve b) 4,4'-bipiridin organik ligandlarının, c) bileşiğin görünür bölge yayma spektrumları.

\section{Tartıșma ve Sonuç}

Yeni iki boyutlu 2-süfoterfitalik asit ve 4,4'-bipiridin içeren koordinasyon bileşiği, $\left\{\left(\mathrm{H}_{3} \mathrm{stp}\right)\left(4,4^{\prime}-\text {-bipy }\right)_{2} .\left(\mathrm{H}_{2} \mathrm{O}\right)\right\}_{\mathrm{n}}$, hidrotermal yöntem kullanılarak sentezlenmiştir. Sentezlenen yeni bileşik yapısal olarak karakterize edilmiş ve bu bileşiğin soğurma ve fotolüminesans özelliği katı formda oda sıcaklığında ve görünür bölge de araştırılarak bileşiği oluşturan serbest organik ligandlar ile karşılaştırılmıştır. Bileşiğin kristal yapısı büyütüldüğünde, 2-sülfoterfitalik asit ve 4,4'-bipiridin grupları arasındaki güçlü $\mathrm{O}-\mathrm{H} \cdots \mathrm{N}$ hidrojen bağları ile yapı bir boyutlu sonsuz zincirleri oluşturmaktadır. Oluşan bir boyutlu zincir yapılarla birlikte $\mathrm{C}-\mathrm{H} \cdots \mathrm{O}$ hidrojen bağları ve aromatik halkalar arasındaki $\pi-\pi$ etkileşimleri iki-boyutlu tabakalı yapı şeklinde büyümektedir.

Bileşiğin fotolüminesans spektrumu incelendiğinde görünür bölgede şiddetli yeşil-sarı renkte geniş yayma bandı gözlenmiştir. Bileşik ve bileşiği oluşturan organik ligandlar karşılaştırıldığında yayma bantlarının maksimum dalgaboylarında kırmıza kayma gözlenmiştir. Böylece, bileşik sarımsı-yeşil 1şık yayar. Bileşik, \{( $\left.\mathrm{H}_{3} \operatorname{stp}\right)(4,4$ 'bipy $\left.)_{2} \cdot\left(\mathrm{H}_{2} \mathrm{O}\right)\right\}_{\mathrm{n}}$, hem teknolojik hem de endüstriyel üretimde yapısal bir ara malzeme olarak kullanılabilir.

\section{Ek Veriler}

Bu makalede verilen kristalografik veriler, Cambridge Crystallographic Data Centre'da depolanmış olup talep üzerine, (The Director, CCDC, 12 Union Road, Cambridge, CB2 1EZ, UK; e-mail: deposit@ccdc.cam.uk; www: 
Nevşehir Bilim ve Teknoloji Dergisi (2019), 8(2) 103-113

http://www.ccdc.cam.ac.uk; fax: +44 1223 336033) CCDC 1896941 Nolu depolama numarası ile ücretsiz olarak temin edilebilir.

6. Kaynaklar

[1] Coban, M. B., Synthesis and photophysical properties of new Eu(III) complex, Balıkesir Üniversitesi Fen Bilimleri Enstitüsü Dergisi, 19, 7-15, 2017.

[2] Kara, H., Synthesis and 3D crystal structure of new Ni(II)-ion-pair complex, Balıkesir Üniversitesi Fen Bilimleri Enstitüsü Dergisi, 20, 1-7, 2018.

[3] Shahi, P.K., Kumar, B., Prakash, R. ve Rai, S.B., Investigation of optical properties and energy transfer in Eu(III) and $\mathrm{Tb}(\mathrm{III})$ based composite compound dispersed in polar, non-polar solvents and polymer matrix, Materials Research Express, 6, 046204, 2019.

[4] Bolligarla, R., Durgaprasad, G. ve Das, S.K., Coordination polymers from dithiolato complexes and alkali metal cations: How a crystallizing and coordinating solvent influences the dimensionality, Inorganica Chimica Acta, 486, 412-424, 2019.

[5] Tian, Y., Allan, P.K., Renouf, C.L., He, X., McCormick, L.J. ve Morris, R.E., Synthesis and structural characterization of a single-crystal to single-crystal transformable coordination polymer, Dalton Transactions, 43,1519-1523, 2014.

[6] Wei, X.-H., Yang, L.-Y., Liao, S.-Y., Zhang, M., Tian, J.-L., Du, P.-Y., Gu, W. ve Liu, X., A series of rare earth complexes with novel non-interpenetrating 3D networks: synthesis, structures, magnetic and optical properties, Dalton Transactions, 43, 5793-5800, 2014.

[7] Giricheva, N.I., Syrbu, S.A., Bubnova, K.E., Fedorov, M.S., Kiselev, M.R. ve Girichev, G.V., H-complexes in the "4-n-alkoxybenzoic acid: 4-pyridyl 4'-n-alkoxybenzoate” system. IR spectroscopy and quantum chemical calculations, Journal of Molecular Liquids, 277, 833-842, 2019.

[8] Kurban, M. ve Gündüz, B., Study of structural, optical properties and electronic structure of PTCDI-C5 organic nanostructure, Chemical Physics Letters, 691, 14-21, 2018.

[9] Gündüz, B. ve Kurban, M., Photonic, spectroscopic properties and electronic structure of PTCDI-C8 organic nanostructure, Vibrational Spectroscopy, 96, 46-51, 2018.

[10] Deng, Y., Yao, Z.-Y., Wang, P., Zhao, Y., Kang, Y.-S. ve Sun, W.-Y., Metal organic frameworks with 1,3-bis(1imidazolyl)-5-(imidazol-1- ylmethyl)benzene and 3,3'-disulfobiphenyl-4,4'-dicarboxylate ligands: Synthesis, structure and selectively sensing property, Sensors and Actuators B Chemical, 244, 114-123, 2017.

[11] Oylumluoglu, G., 4,4- bipiridin ve 2-amino-1,4-benzendikarboksilik asit tabanlı yeni bileşiğin sentezlenmesi ve hidrojen bağlı 3D polimerik yapısı, Balıkesir Üniversitesi Fen Bilimleri Enstitüsü Dergisi, 20, 320-326, 2018.

[12] Wang, J., Cai, J.-M., Wang, A.-Y., Huang, B.-F., Xiao, H.-P., Li, X.-H. ve Morsali, A., Synthesis, crystal structures and photoluminescence properties of two silver(I) coordination polymers with nano size channels based on 2-sulfoterephthalic acid ligand, Inorganica Chimica Acta, 394, 466-471, 2013.

[13] Xiao, S.-S., Zheng, X.-J., Yan, S.-H., Deng, X.-B. ve Jin, L.-P., Architecture of europium complexes with sulfobenzenedicarboxylates, Crystal Engineerıng Communications, 12, 3145-3151, 2010.

[14] P.-Y. Du, W. Gu, X. Liu, A three-dimensional Nd(III)-based metal-organic framework as a smart drug carrier, New Journal of Chemistry, 40, 9017-9020, 2016.

[15] Døssing, A., Kadziola, A., Gawryszewska, P., Watras, A. ve Melchior, A., Structure, stability and spectroscopic features of the neodymium(III) complex of the octadentate polypyridine ligand 6,6'-bis[bis(2pyridylmethyl)aminomethyl]-2.2'-bipyridine, Inorganica Chimica Acta, 467, 93-98, 2017.

[16] Coban, M.B., Hydrothermal synthesis, crystal structure, luminescent and magnetic properties of a new mononuclear Gd III coordination complex, Journal of Molecular Structure, 1162, 109-116, 2018.

[17] Oylumluoglu, G., Coban, M.B., Kocak, C., Aygun, M. ve Kara, H., 2-and 1-D coordination polymers of Dy(III) and Ho(III) with near infrared and visible luminescence by efficient charge-transfer antenna ligand, Journal of Molecular Structure, 1146, 356-364, 2017.

[18] Coban, M.B., Kocak, C., Kara, H., Aygun, M. ve Amjad, A., Magnetic properties and sensitized visible and NIR luminescence of Dy III and Eu III coordination polymers by energy transfer antenna ligands Magnetic properties and sensitized visible and NIR luminescence of Dy III and Eu III coordination polymers by ene, Molecular Crystals and Liquid Crystals, 648, 202-215, 2017.

[19] CrysAlisPro Software System, Version 1.171.38.43, Rigaku Corporation, Oxford, UK, 2015.

[20] Sheldrick, G.M., A short history of SHELX, Acta Crystallographica Section A Foundations of Crystallography, 
64, 112-122, 2008.

[21] Dolomanov, O. V., Bourhis, L.J., Gildea, R.J., Howard, J.A.K. and Puschmann, H., OLEX2 : a complete structure solution, refinement and analysis program, Journal of Applied Crystallography, 42, 339-341, 2009.

[22] Spek, A.L., Structure validation in chemical crystallography, Acta Crystallography Section D: Biological Crystallography, 65, 148-155, 2009.

[23] Kocak, C., Oylumluoglu, G., Donmez, A., Coban, M.B., Erkarslan, U., Aygun, M. ve Kara, H., Crystal structure and photoluminescence properties of a new monomeric copper(II) complex: bis(3-\{[(3hydroxypropyl)imino]methyl\}-4-nitrophenolato- $\kappa^{3}$ O,N,O')copper(II), Acta Crystallographica Section C, 73, 414-419, 2017.

[24] Erkarslan, U., Donmez, A., Kara, H., Aygun, M. ve Coban, M.B., Synthesis, Structure and Photoluminescence Performance of a New Er3+-Cluster-Based 2D Coordination Polymer, Journal of Cluster Science, 29, 11771183, 2018.

[25] Zhou, X., Zhao, X., Wang, Y., Wu, B., Shen, J., Li, L. ve Li, Q., Eu(III) and Tb(III) Complexes with the Nonsteroidal Anti-Inflammatory Drug Carprofen: Synthesis, Crystal Structure, and Photophysical Properties, Inorganic Chemistry, 53, 12275-12282, 2014.

[26] Kara, D.A., Donmez, A., Kara, H. ve Coban, M.B., Structural and spectroscopic characterization of a new

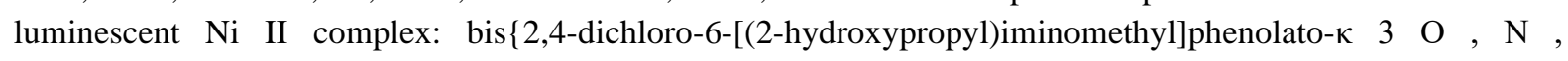
O '\}nickel(II), Acta Crystallographica Section C, 74, 901-906, 2018.

[27] Coban, M.B., Magnetic and photophysical properties of new TbIII -based two-dimensional hydrogen-bonded polymer, Journal of Molecular Structure, 1177, 331-337, 2019.

[28] Coban, M.B., Erkarslan, U., Oylumluoglu, G., Aygun, M. ve Kara, H., Hydrothermal synthesis, crystal structure and Photoluminescent properties; 3D Holmium(III) coordination polymer, Inorganica Chimica Acta, 447, 8791, 2016.

\section{Extended Abstract}

\section{Introduction}

Over the last two decades, coordination polymers (CPs) have recently attracted strong interest from scientific environments, due to their fascinating coordination architectures and potential applications, such as molecular recognition, luminescence, catalysis, gas adsorption and separation processes, ion exchange, nonlinear optics and etc [1$6]$.

In the most instances, O-donor carboxylic acids and N-donor ligands are favored since the constructed coordination compounds usually have potential applications and relatively high stability [7,8]. The 2-sulfoterephthalic acid and carboxylic acid derivatives include two carboxylates and one sulfate group, which could be useful in different dimensional and versatile architectures construction of new coordination polymers. Besides, the $\mathrm{SO}_{3}$ group, which is mostly regarded as weakly linkage, can make its coordination ability more sensitive and flexible to the chemical environment, which is an origination of greater coordination versatility [9,10].

However, the few kinds of coordination compounds is studied in some report due to their biomedical applications, their fascinating architectures, good biocompatible, permanent high and regular porosity, and drug molecule storage and delivery versatile functionality and. Also, in the coordination polymer-drug systems, interactions such as hydrogen bonds, $\pi-\pi$ and electrostatic interaction govern the release and of diffusion drug, which is remarkable to achieve controllable and prolong drug release [11,12].

In recent years, our research group have actively studied in the structural and optical characterization of the organic molecules, which is include various aromatic carboxylate and pyridine derivatives [13-15]. In this study, the new coordination compound, $\left\{\left(2-\mathrm{H}_{3} \mathrm{stp}\right)(4,4 \text { '-bipy })_{2} .\left(\mathrm{H}_{2} \mathrm{O}\right)\right\}_{\mathrm{n}}$, $\left(2-\mathrm{H}_{3} \mathrm{stp}=2\right.$-sulfoterephthalic acid, 4,4'-bipy=4,4'-bipiridine $)$, was synthesized as a continuation of our previous studies and characterized by the single crystal X-ray diffraction method. Intra- and intermolecular $\mathrm{O}-\mathrm{H}^{\cdots} \mathrm{N}, \mathrm{O}-\mathrm{H}^{\cdots} \mathrm{O}$ and $\mathrm{C}-\mathrm{H}^{\cdots} \mathrm{O}$ hydrogen bonds and $\pi-\pi$ interactions were analyzed. Additionally, the solid-state UV-Vis spectra of the compound have been obtained by comparing with its free ligands. The solid-state photoluminescence spectra has been investigated in detailed.

\section{Method}

The chemicals used in the experiments were commercially obtained from Sigma-Aldrich, and were used without any purification. Elemental analyses of complex was carried out with a LECO-CHNS-932. Single-crystal X-ray diffraction data was collected at room temperatıre on a Rigaku-Oxford Xcalibur diffractometer with an EOS-CCD 
Nevşehir Bilim ve Teknoloji Dergisi (2019), 8(2) 103-113

detector using graphite-monochromated MoK $\alpha$ radiation with CrysAlis ${ }^{\text {Pro }}$ software [16]. The crystal structure of the compound was solved in OLEX2 by direct method with SHELXT $[17,18]$. All non-hydrogen atoms were assigned anisotropic displacement parameters and refined without positional constraints. Hydrogen atoms were included in idealized positions with isotropic displacement parameters on strained to 1.2 times the Uequiv of their attached carbon atoms. Details of the supramolecular $\pi$-interactions were calculated with PLATON 1.17 package program [19]. Solid state absorption spectra were recorded on Ocean Optics Maya 2000 Pro UV-vis Spectrometer. Photoluminescence spectrum was obtained by Andor Solis SR 500i BL model spectrometer at room temperature.

\section{Results and Discussion}

The new two-dimensional coordination compound, $\left\{\left(2-\mathrm{H}_{3} \mathrm{stp}\right)(4,4 \text { '-bipy })_{2} \cdot\left(\mathrm{H}_{2} \mathrm{O}\right)\right\}_{\mathrm{n}}$, which is containing 2sulfoterephthalic acid and 4,4'-bipyridine, was synthesized using the hydrothermal method. The new compound synthesized was structurally characterized, uv-vis and photoluminescence properties of this compound were investigated in solid form at room temperature in the visible region and compared with that of its free ligands. In compound, 2sulfoterephthalic acid and 4,4'-bipyridine groups are linked $\mathrm{O}-\mathrm{H} \cdots \mathrm{N}$ strongly hydrogen bonding interactions. These interactions give rise to a $1 \mathrm{D}$ hydrogen bonding chain structure. These $1 \mathrm{D}$ chains connect each other with intramolecular and intermolecular $\mathrm{C}-\mathrm{H} \cdots \mathrm{O}$ hydrogen bonds and face to face $\pi-\pi$ interactions, which form $2 \mathrm{D}$ layer structure. The emission spectrum of the compound indicates strongly yellowish-green a wide band emission in the visible region. The solid state photoluminescence spectra of compound were analyzed in comparison with that of their free ligands. The luminescence band of the compound is observed about at $50 \mathrm{~nm}$ red shift compared with their parent ligand. Thus, the compound emits yellowish-green light. The compound, $\left\{\left(2-\mathrm{H}_{3} \mathrm{stp}\right)(4,4 \text { '-bipy })_{2} \cdot\left(\mathrm{H}_{2} \mathrm{O}\right)\right\}_{\mathrm{n}}$, can be used as a structural intermediate in both technological and industrial production. 